Military Technical College

Kobry Elkobbah,

Cairo, Egypt

May 25-27,2010

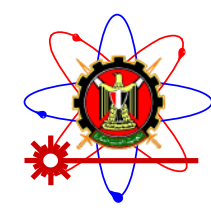

$5^{\text {th }}$ International Conference on Mathematics and Engineering Physics (ICMEP-5)

\title{
PH-23
}

\section{Plasma technology in power engineering}

\author{
Prof Pavel Kolat, Doc.Ing.zdenek kadlec, Prof Vladimir dembovsky.
}

\begin{abstract}
Plasma technology utilised in power engineering can be divided to:

I. High temperature plasma technology. The development of experimental equipment for decomposing the toxic compounds that arise in the pyrolysis or combustion of PCB oils, plastics and other dangerous wastes, by subsequently raising the combustion temperature above the threshold for dissociation of toxic gas molecules. The design of experimental dissociation chamber plant, rated at $100 \mathrm{~kg} /$ hour of dangerous waste is presented.

II. Low temperature plasma technology is one of the possibilities how to replace efficiently noble fuels (mazut or gas) used for the start-up and stabilization of power stations and utilization in the incineration plants. The output $320 \mathrm{kVA}$ of plasma generator and temperature $6000 \mathrm{oC}$ for the boiler is sufficient. The operating costs of plasma ignition of coal powders are significantly lower than the start-up of boiler with mazut, and/or the start-up or stabilization with the use of gas. These conclusions have been fully confirmed and verified.
\end{abstract}

\section{Introduction to the high temperature plasma technology:}

Modern technology of combustion dangerous organic wastes, inclusive chlorates hydrocarbons and other organic halogen compounds rely upon optimization of combustion conditions to ensure higher temperature, longer residence time of the treated materials in high temperature zones and larger oxygen amount in the post-combustion zone, where the combustion products are after burned. The aim is to decompose even the most stable of toxic organic compounds with an efficiency better than $99,9 \%$. Research results indicates that to ensure complete liquidation of chlorates hydrocarbons (PCB), a temperature not lower than $1100{ }^{\circ} \mathrm{C}$ has to be maintained in the secondary combustion stage with the aid of a surplus of oxygen. Oxidation enables the destruction of organic compounds, with exception of $\mathrm{CFCl}$ ( freons - $\mathrm{CFCl}_{3}, \mathrm{CF}_{2} \mathrm{Cl}_{3}, \mathrm{CF}_{3} \mathrm{Cl}$ and chlortrifluorethylene $-\mathrm{C}_{2} \mathrm{ClF}_{3}$ ) and at lower temperature arise also highly reactive radicals $\mathrm{OH}$. Liquidation of the above mentioned dangerous wastes, accompanied with origin of gaseous toxic compounds and liquidation of contingent chemical military weapons based on organic compounds, which are so stockpiled a precluded places, is not in ordinary furnaces viable. This temperature can be obtained by use of high temperature plasma technique. Advantages of plasma technology [1] for treating toxic gaseous compounds are:

1. Highly effective decomposition of toxic compounds in the gaseous products of pyrolysis or combustion processes. 
2. Prevention of recombination reaction which would convert simple molecules or atomic particles back into initial PCB, PCDD, PCDF or other high molecular organic compounds from which those particles arose.

3. The products of alternative pyrolysis liquidation can arise energetically usable fuel gas with high content of $\mathrm{CO}, \mathrm{H}_{2}$ and hydrocarbons.

4. Much lower capital outlays than are required for incinerating plants using an active coke filter and a reducing atmosphere.

In contradiction to existing plasma techniques, enabling liquidation of toxic compounds, inclusive these in military chemical weapons, however the equipment envisaged in the present project also exploits the radiation effect of long plasma beams, with temperatures up to some $20000{ }^{\circ} \mathrm{C}$ which destabilizes molecules of toxic compounds by ultraviolet radiation at frequencies concentrated around $10^{14} \mathrm{~Hz}$. Molecules excited to higher potential energies by radiation effects of thermal plasma are less stable, and decompose at lower temperatures.

\section{The goal of the project:}

The goal is a design of a plasma dissociation chamber with $3 \times 1$ MVA for incineration plants, combusting dangerous wastes. The design will utilize experimental

Figure (1): Module with 3 plasma torches and cooler.

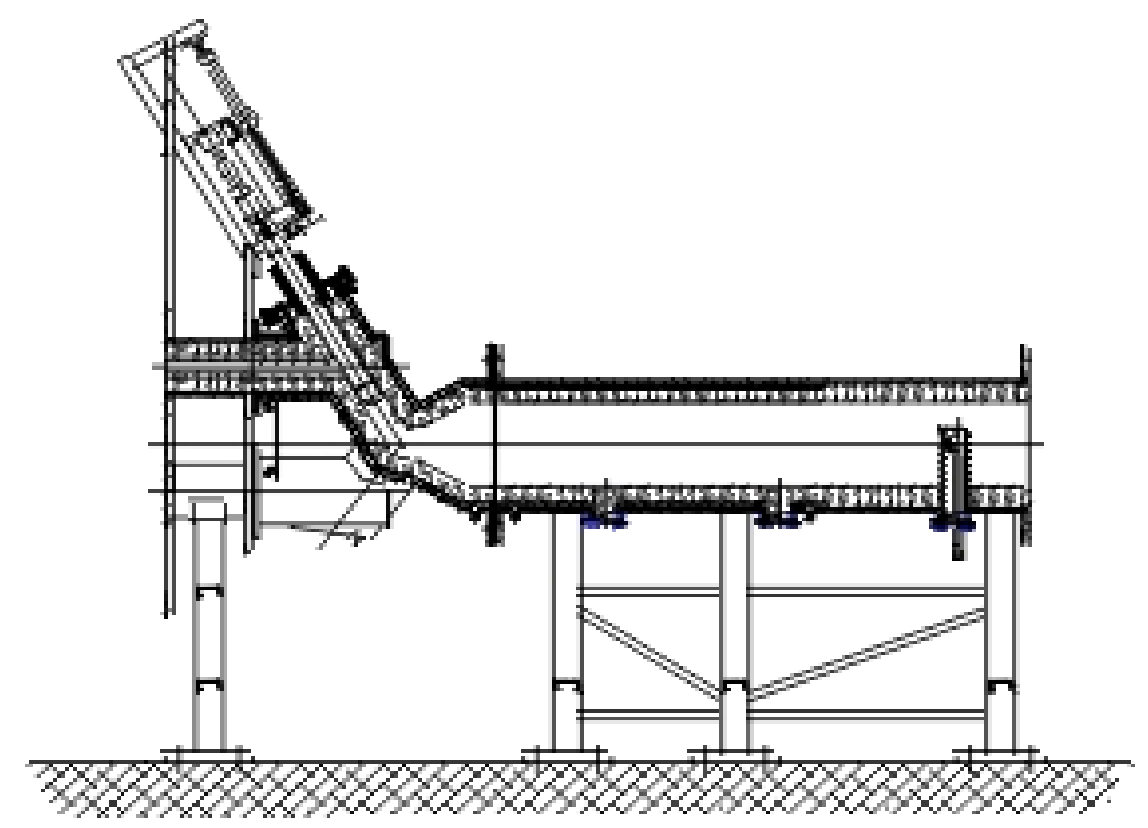




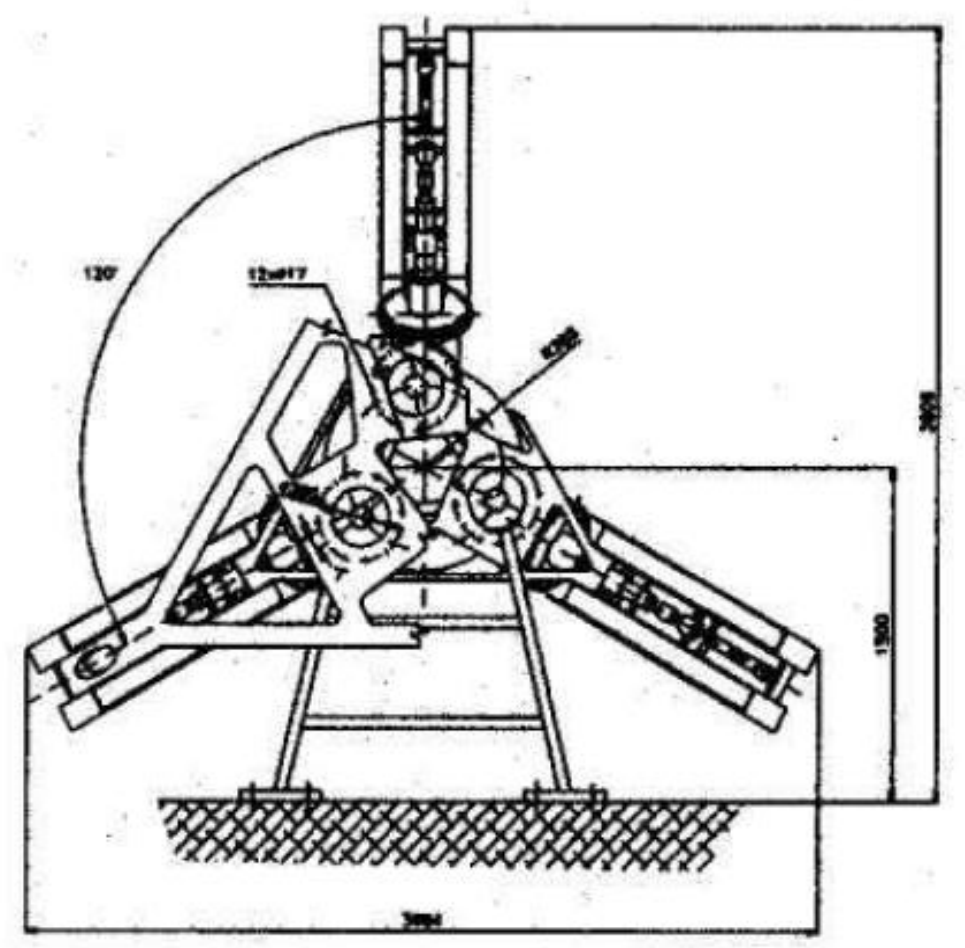

results, obtained from present operating plants, results of numerical simulation by means of program Fluent and development results of thermal plasma arc generators 1 MWe output, gained on metallurgy workplace. Module, including plasma dissociation chamber and cooling device Figure (1) for freezing unobjectionable dissociation products should be usable for incineration plants with output to $100 \mathrm{~kg}$ per hour, eventually even for mobile devices, which neutralize wastes, containing PCB, PFC and CFC compounds.

Dissociation chamber is designed with a water-cooled jacket and refractory lining to allow operation with flue gas temperature to $1700^{\circ} \mathrm{C}$. Figure (2). The chamber is equipped with three plasma burners maximum output of $3 \times 1 \mathrm{MWe}$. Start and control arc length will allow the hydraulic system of synchronized axial movement of the "Master and Slave" with the current regulator, enabling a smooth change of power in the range 0 to $100 \%$. The chamber is designed for use in a hazardous waste incinerator of $100 \mathrm{~kg} / \mathrm{hr}$ at an average heating value of waste $20 \mathrm{MJ} / \mathrm{kg}$. The reverse recombination of dissociated compounds where there are combustion gases from the cooling temperature of $1600{ }^{\circ} \mathrm{C}$ at a temperature of $480 \div 500{ }^{\circ} \mathrm{C}$ for a period of less than $0.3 \mathrm{sec}$. This is the main condition for construction of the cooler.

Technical University of Ostrava provides a solution for the project laboratory plasma technology with a power transformer station 1 MVA, thyristor power regulator Variant with water and gas economy and documentation for patent-protected design of plasma torch Prof. Dembovsky [2,3]. The solution proposed project requires the addition of dedicated laboratory reactors (inductors) with a switchboard 1 MVA, combustion chamber equipped with a burner, dissociation chamber, flue gas fan, three plasma torches and air conditioning device for exhaust toxic fumes from the experimental work. 


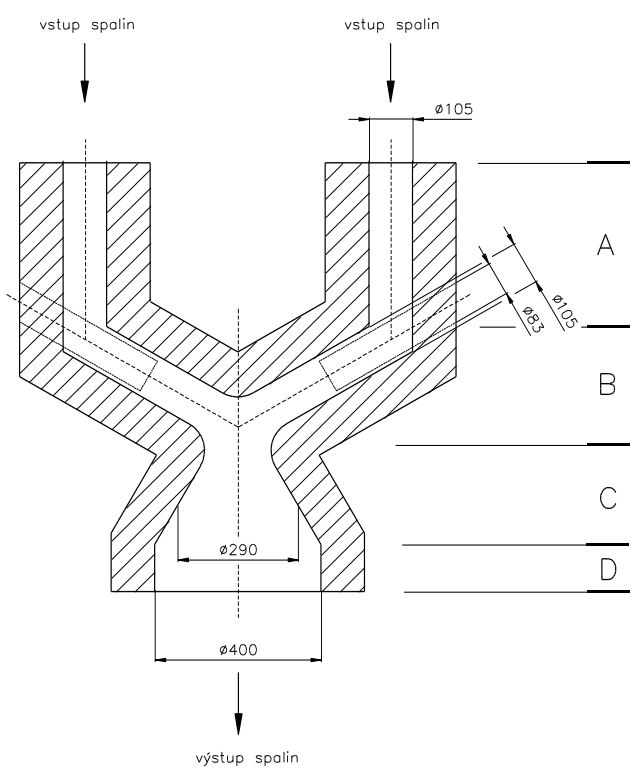

Figure (2): Scheme of the dissociation chamber

\section{Modelling temperature and velocity fields:}

For modelling was used program FLUENT. This program is based on solving Navier-Stokes equations of viscous flow including the effects of turbulence, heat transfer, radiation and chemical reactions. The solution is network of finite volumes, which is

determined by the discreditation. Modelling tasks have been simplified for a solution in 2D geometry. The physical network was created from drawings. Other physical quantities are awarded in accordance with the specified and calculated values. Flue gas temperature at the inlet to the chamber was added to the examples $200^{\circ} \mathrm{C}$ and velocity $20 \mathrm{~m} . \mathrm{s}^{-1}$. Gas input was modelled using the input rotation speed by an angle of $45^{\circ}$ from the axis of entry. Other parameters were awarded in accordance with the calculations made.

\section{Conclusion:}

The aim of the project is the construction of the module chamber high temperature plasma dissociation of the electrical power $3 \times 1$ MVA incineration of hazardous wastes using experimental results obtained at the same time operated incinerators [4], the results obtained by numerical simulation program FLUENT, and the results of the development of thermal plasma arc generators from metallurgy. The project is to determine the conditions and cooler design for "freezing" of products and prevent backward dissociation recombination of simple molecules, radicals and atomic particles on the initial toxic compounds. The strategic aim of the project is preparing materials for the manufacture of a prototype module for removing toxic compounds from the exhalation of small hazardous waste incinerators.

\section{Introduction to the low temperature plasma technology:}

At present it is possible to specify general trends of utilization of low temperature plasma technology in power engineering [5] :

- Mazut-free boilers starting-up.

- Flame stabilization from coal powder.

- Stabilization of slag outflow from melting boilers.

- Reduction of losses caused by poor burning, e.g. due to coarse crushing.

- Reduction of emissions of nitrogen oxides.

- Waste incineration plants. 
The plasma generator consists of two axially positioned mutually insulated copper electrodes - cathode and anode comprising the axially symmetrical discharge chamber. The air creating the plasma enters through two inlets into the discharge chamber. Under the influence of aerodynamic forces a part of the arc in the vicinity of electrodes moves on the surface of electrodes and the remaining part of arc is stabilized in the axis of the discharge chamber. Parts of plasma generator exposed to effects of heating flow created by the electric arc are cooled with water. Plasma generators can be produced in different output series and their output is preliminary determined according to a type and quantity of combusted mixture. One of basic properties of coal affecting a choice of plasma generator is the content of volatile combustible matter.

\section{Processes at contact of primary mixture with low-temperature plasma:}

- Temperature of primary mixture on inlet is approximately $80-150{ }^{\circ} \mathrm{C}$.

- At contact of the flow of cold air mixture with plasma torch the ionised gas flows out from the nozzle of plasma generator while simultaneously heats up the air and coal particles.

- Temperature of electric arc of plasma on the outlet from plasma generator is 2000 $5000^{\circ} \mathrm{C}$, thus a great temperature gradient occurs when the plasma arch is mixed with the relatively cold mixture of coal powder and air.

- $\quad$ Air in the primary mixture is heated up from a plasma source in accordance with its thermal output and the difference of temperatures of plasma and air.

- In a zone of mutual efficiency of plasma with primary mixture a coal particle with medium dimension of $100 \mu \mathrm{m}$ heats up at velocity of $1000-10000^{\circ} \mathrm{C} / \mathrm{sec}$. It was found out experimentally that coal particles with dimensions up to $0.25 \mathrm{~mm}$ disintegrate by the effect of thermal stress to the tenth of its size. This phenomenon called also elsewhere as a thermal explosion of particles leads to a vehement growth of the surface within the boundary of gaseous and solid phase and subsequently to the increased reaction capability of fuel.

- Thermal shock Figure (3), accelerates the release of combustible substances many times due to the significantly enlarged and disturbed surface and due to the occurrence of very fine particles. Volatile substances are released from these particles such as $\mathrm{CO}, \mathrm{CO}_{2}, \mathrm{CH}_{4}$, $\mathrm{C}_{6} \mathrm{H}_{6}, \mathrm{~N}_{2}$, water and nitrogen retaining compounds (pyridine $\mathrm{C}_{5} \mathrm{H}_{5} \mathrm{~N}$, pyrrole $\mathrm{C}_{4} \mathrm{H}_{5} \mathrm{~N}$ ). A vehement growth within the boundary of gaseous and solid phase leads afterwards to the increased reaction capability of fuel.

Destruction of ash compounds and reaction of volatile substances with oxygen occurs, whilst the following elements are created in the gaseous phase:

- $\quad$ Atomic forms $(\mathrm{O}, \mathrm{H}, \mathrm{N}, \mathrm{C}, \mathrm{S})$

- $\quad$ Elements of mineral parts of coal ( $\mathrm{Si}, \mathrm{Al}, \mathrm{Ca})$

- $\quad$ Radicals $(\mathrm{NH}, \mathrm{CH}, \mathrm{CN}, \mathrm{OH}$ and other)

- $\quad$ Positive ions $\left(\mathrm{C}^{+}, \mathrm{H}^{+}, \mathrm{N}^{+}, \mathrm{O}^{+}, \mathrm{CO}^{+}, \mathrm{Si}^{+}, \mathrm{K}^{+}\right.$and other

- $\quad$ Negative ions $\left(\mathrm{O}^{-}, \mathrm{H}^{-}, \mathrm{N}_{2}^{-}\right)$

- $\quad$ The so called electron gas occurs also in the gaseous phase.

Afterwards a coke residue is heated up to the temperature of gasification and a coke residue reacts with the forming gaseous phase.

With the transition of volatile substances such as $\mathrm{CO}, \mathrm{H}_{2}, \mathrm{CH}_{4}, \mathrm{C}_{6} \mathrm{H}_{6}, \mathrm{CO}_{2}, \mathrm{~N}_{2}, \mathrm{H}_{2} \mathrm{O}$ and other substances into the gaseous phase they begin to react with the air and also with each other. [2]. 

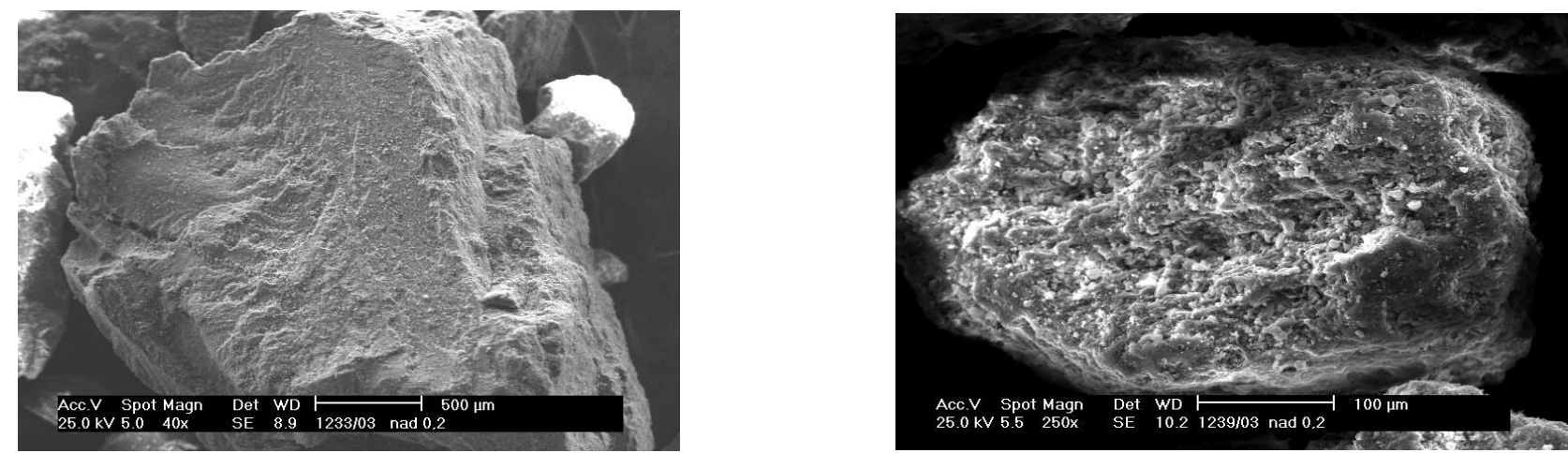

Figure 3: Coal particles before and after the contact with low-temperature plasma

\section{Application of plasma technology at the Power Engineering:}

The boiler at power station is a bi-draught type with granulating furnace with total thermal output of $290 \mathrm{MWt}$ and steam output of $350 \mathrm{t} / \mathrm{h}$. The boiler incorporates five grinding circuits with direct blowing of powder into 5 two-part burners positioned above the discharge hopper of the combustion chamber. Main burners are designed as turbulent, waste and flow burners. Before the performance of general starting-up tests with the boiler without any use of other noble fuels, first the generator of lowtemperature plasma was subjected to many tests, its characteristics were measured and output control was tested. Figure (4).

Figure (4): Test of low-temperature plasma generator and activation of coal powder
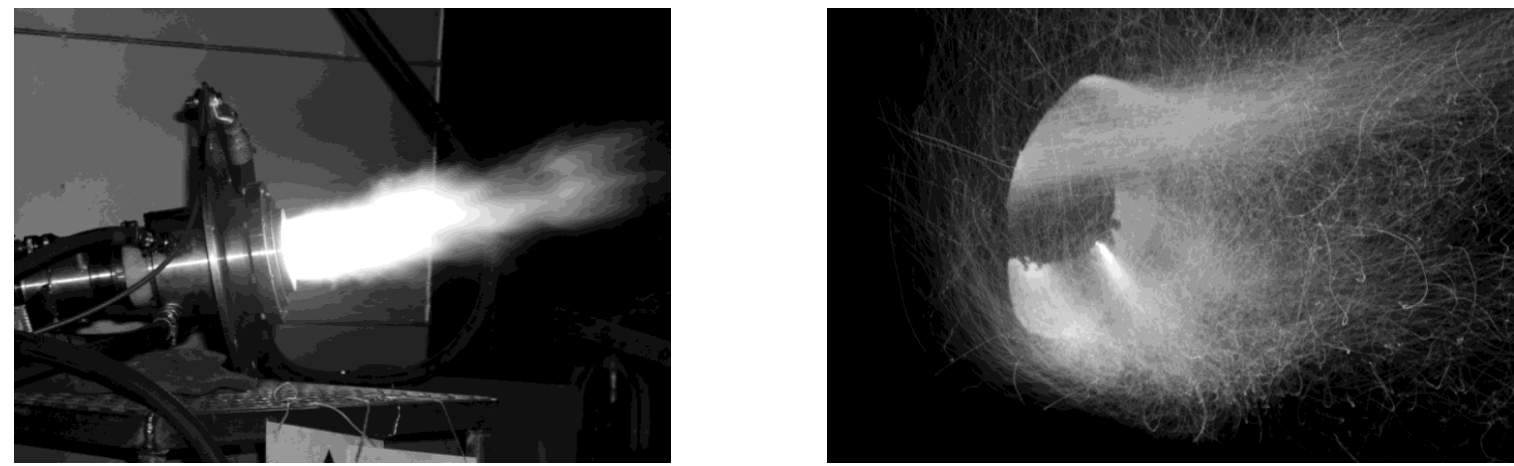

It was succeeded to shorten significantly the starting times by plasma technology. Time saving varied from $30-45$ minutes in comparison with mazut starting-up. At the same time the corresponding operation costs in connection with heating up the boiler were reduced.

Look into the combustion chambers at boiler starting-up by means plasma technology without the use of any other noble fuel is in Figure (4).

Modelling of flow in the generator of low-temperature plasma

In the first phase the mathematical model was defined for the air only. At present the flow is modelled for two general modes: working mode and operation mode. The working mode simulates the operation of a plasma burner at present, but without a heat source, i.e. for the case when the discharge between anode and cathode has not taken place yet. The operation mode simulates a rest condition of the boiler, i.e. the case when the boiler is stabilized and the air flows through the plasma generator with minimum quantity, which avoids the penetration of coal powder into the plasma generator. So, the general goal is the study of behaviour of plasma air in the plasma generator and potential optimising this setting-up from the viewpoint of operation properties. 
The computation region was prepared on the basis of the existing drawings. The particular plasma burner consists of two turbulators and outlet diffuser zone of the burner. Points and lines are situated in the computation region serving for evaluation of the investigated quantities and profiles.

The main goal was to describe and visualize phenomena that come into existence when two air flows mix in the region of cathode. The other outcome was velocity profiles in the outlet region, the other additional evaluation was based on the so called flow lines that describe trajectories of mass less particles, i.e. liquid molecules. The first case investigated the flow of gas without thermal expansion caused by electric discharge.

At present a mathematical model of flowing with heat generation is being prepared. A region in the shape of electrical discharge was defined in geometry. The description of the origin of plasma is problematic. At present the flowing medium is the air, because the disintegration of molecules is very difficult to describe and at the same time there does not exist the general CFD program, which would include a model for these specific technical problems. The only possibility is to supplement the commercial model with the user defined models written in the language $\mathrm{c}++$, fortran, etc. Significant problems at modelling of flow with such a high temperature are material properties. Air properties were known up to $3000^{\circ} \mathrm{C}$ and for higher temperatures the values were extrapolated. The computation model should also include radiation, which cannot be neglected at such high temperatures. The radiation model was increasing the heat dissipation in inner parts of plasma generator and with elimination of this model the temperatures reached unrealistic values, which was caused by the relatively low conductivity of the air. This model will serve for simulation of starting-up the boiler and potential optimising this process. Figure $(5,6)$.
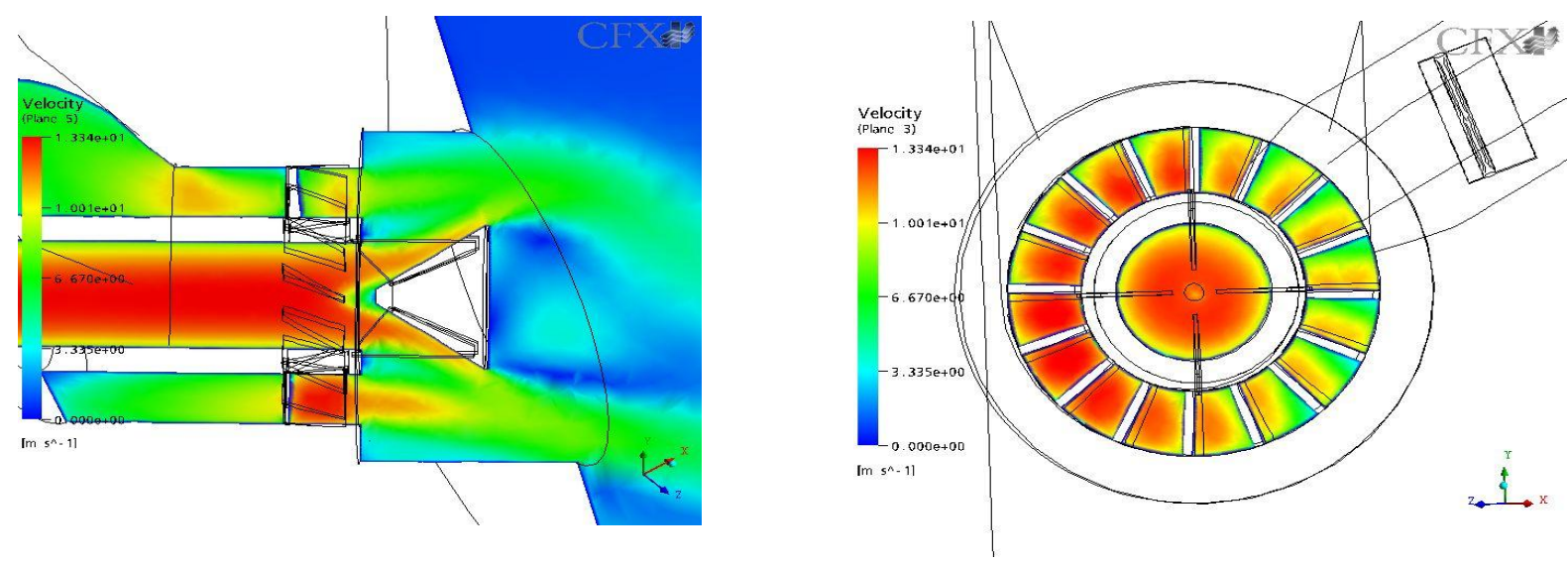

Figure (5): Illustration of medium velocity in longitudinal section for turbulent burner

Figure (6): Illustration of trajectories of coal particles in the field of turbulent burner 


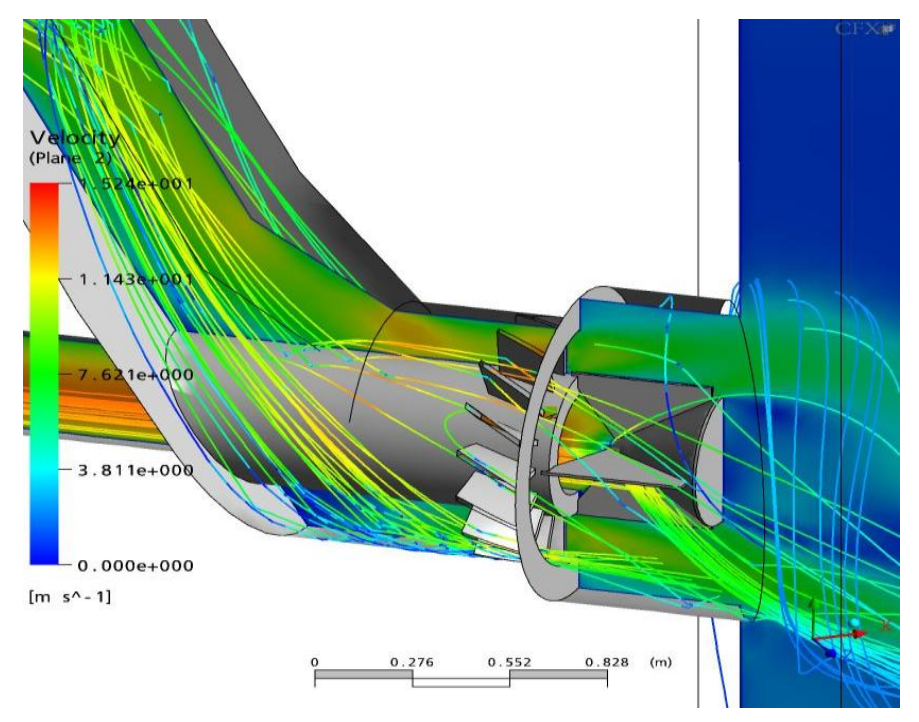

\section{Conclusion for low temperature plasma technology:}

The so far results show the suitability and perspective applications of plasma ignition and stabilization burners. Today the system has been automated and it can be easily operated directly from the control room. The implementation of this type of plasma technology in the individual coal blocks has been already proved in two general modifications. There are the boilers with a direct blowing of coal powder into the combustion chamber and the boilers with a bunker with coal powder.

At present the operation conditions are constantly optimised to achieve a longer service life of electrodes and to ignite the primary mixture and start up of the boiler as soon as possible, thereby saving fuel consumption.

Operation costs for plasma ignition of coal powders are significantly lower that the operation with mazut starting-up of boiler and/or starting or stabilization with gas. These conclusions have been fully confirmed and verified. Project solutions may results in savings of fossil fuels used for setting boilers alight. Also other financial savings, boilers' longer operational life, emission decreases, as well as other positive effects may be expected.

The application of the project results by some coal-fired power stations in the Czech Republic and in the Poland may imply an effect of multiplicity.

\section{References:}

[1] Camacho, S.L.-Dembovský,V.and Gonzales, V. et al.: Arc plasma processes. UIE Paris, 1988.

[2] Patent PV 738/92. Vítkovice, a.s. Dembovský, V.- Motloch, Z.- Golonka, A.: High temperature plasma generator . 1992.

[3] Patent 241 795. VŽKG Ostrava. Dembovský, V.: Coaxial plasma burner. 1984.

[4] Kolat, P. a kol.: Liquidation of radioactive waste by combustion. Prototype of incineration plant for low level radioactive wastes. State research project A-01-159812/5. VÚJE Trnava, VŠB Ostrava, 1990-94.

[5] Kapenko, E.I. a kol.: Plasma start up systems in Russian power stations Novosibirsk, Snaukas PAH 1998. 